\title{
Levobupivacaine combined with dexamethasone for serratus plane block can provide long-lasting analgesia in multiple rib fractures
}

\author{
Santi Di Pietro, ${ }^{1}$ Benedetta Mascia, ${ }^{2}$ Stefano Perlini, ${ }^{3}$ Giorgio Antonio Iotti ${ }^{2}$ \\ ${ }^{1}$ Emergency Medicine Fellowship Program; ${ }^{2}$ Servizio Anestesia e Rianimazione $2 ;{ }^{3}$ Clinica Medica 2, \\ Department of Internal Medicine, San Matteo Hospital, University of Pavia, Pavia, Italy
}

\begin{abstract}
Serratus plane block (SPB) is a technique of regional anesthesia that has recently been proposed to treat pain in rib fractures. In this context, SPB is currently performed using a variety of local anesthetics at different concentrations and dilutions, reporting a duration of pain control up to 12 hours following the procedure. To the best of our knowledge, the addition of dexamethasone to the local anesthetic for this specific regional block has never been documented. Here we report three patients that were treated for multiple rib fractures with SPB, which included combined dexamethasone and levobupivacaine. Interestingly, the obtained pain control lasted between 23 to 45 hours following the block, thus helping to minimize the opioid use in our patients.
\end{abstract}

\section{Introduction}

Serratus plane block (SPB) is a novel technique of regional anesthesia able to provide analgesia to the lateral region of the thoracic wall. Since its first description in 2013 by Blanco et al., ${ }^{1}$ this technique has been reported for pain control in a variety of clinical

\footnotetext{
Correspondence: Santi Di Pietro, Emergency Medicine Fellowship Program, San Matteo Hospital, University of Pavia, Pavia, Italy. Tel.: +39.0382.502356.

E-mail: santi.dipietro01@universitadipavia.it

Key words: Serratus plane block; Rib fractures; Nerve blocks; Steroids in nerve blocks.

Contributions: SDP and BM were responsible for the clinical management of the treated patients and for the preparation of this manuscript; SP and GI revised the manuscript draft.

Conflict of interest: the authors declare no potential conflict of interest.

Funding: none.

Received for publication: 23 July 2018.

Revision received: 3 November 2018.

Accepted for publication: 9 November 2018.

This work is licensed under a Creative Commons Attribution 4.0 License (by-nc 4.0).

(C) Copyright S. Di Pietro et al., 2019

Licensee PAGEPress, Italy

Emergency Care Journal 2019; 15:7716

doi:10.4081/ecj.2019.7716
}

scenarios, including post thoracotomy pain syndrome, contraction of the latissimus dorsi, post-mastectomy pain syndrome and rib fractures. ${ }^{2-4}$ The latter probably represents the most promising application of the procedure, particularly in the Emergency Department, where rib fractures are frequently encountered and a prompt pain control must to be provided in order to prevent complications like pneumonia and respiratory failure. Under this respect, SPB could be a safer and easier alternative to other regional anesthetic techniques, such as intercostal and paravertebral blocks or epidural, that remain uncommon in the ED as they are time-consuming and hold significant risks. ${ }^{5}$

We report three cases of SPB performed in multiple rib fracture patients using $30 \mathrm{~mL}$ of $0.25 \%$ levobupivacaine diluted with $8 \mathrm{mg}$ dexamethasone. Pain control in our patients lasted twenty-three to fourty-five hours following the block, thus making an opioid-sparing or even an opioid-free pain treatment possible.

In all three cases, the anesthetic solution was injected under sonographic guidance in the fascial plane just superficial to the serratus muscle. The technique is shown on a healthy volunteer in Figures 1 and 2.

\section{Case Reports}

\section{Case \#1}

A 21-year-old man was admitted to our ED after he fell from his motorcycle during a motocross competition, resulting in left 611 rib fractures. Moreover, chest CT-scan also showed severe bilateral pulmonary contusion, mostly affecting the left lung inferior lobe, with concomitant mild pneumothorax.

Patient's vitals were stable. He reported moderate pain at rest, becoming excruciating during motion or deep inspiration (Numerical Rating Scale - NRS - 4-10; the first value indicates rest pain, whereas the second one refers to dynamic pain). The SPB procedure was rapidly effective, with a significant improvement of pain (NRS 1-5) at 15 minutes. One hour later, the patient only reported minimal discomfort (NRS 1-3). Such a satisfactory pain control was maintained for 23 hours, at the time when he requested for the first time rescue analgesia.

Since pain was adequately controlled by NSAIDs and paracetamol during the following days of hospitalization in the Pneumology ward, there was no need to prescribe opioids. The patient was discharged after 7 days of an uneventful hospital stay.

\section{Case \#2}

A 51-year-old male polytrauma patient was diagnosed with left 3-5 rib fractures and concomitant ipsilateral pneumothorax, as well as bilateral shoulder fractures and multiple meniscal tearing of the left knee. He reported severe chest pain at rest that was further exacerbated with movements (NRS 8-10). Moreover, he was unable to cough due to excessive pain. Pain was considerably 
improved 15 minutes after SPB (NRS 2-3), and he could cough without significant discomfort. One hour after the procedure, he reported no pain at rest and only a minimal discomfort when coughing (NRS 0-1). Accordingly, i.v. remifentanyl was discontinued, and the patient was admitted to the ICU after pneumothorax drainage. SPB allowed to achieved a satisfactory 36-hour pain control. During the subsequent 15-day hospital stay he was treated with paracetamol, NSAIDs and tapentadole, the main complaint being shoulder and knee pain. On a single occasion, he required eptadone for the exacerbation of knee pain.

\section{Case \#3}

A 56-year-old male was referred to our ED for a motorbike fall. A CT-scan showed 2-9 left rib fractures with an underlying pneumothorax, concomitant with ipsilateral clavicle and sternum fractures. During the night, he was admitted to the ICU where a chest drainage was placed in the left $5^{\text {th }}$ intercostals space on the midaxillary line. Despite i.v. NSAIDs, paracetamol and remifentanyl, he reported significant chest pain at rest, exacerbated by coughing (NRS 6-8). Therefore, SPB was performed, leading within 15 minutes to a marked decrease of pain both at rest and while coughing (NRS 2-3). Since pain control was maintained one hour after the block, iv remifentanyl dosage could be halved.

After 24-hours, an additional anterior chest drainage was placed due to persistence of pneumothorax: the patient experienced moderate periprocedural pain, resolving within few hours. He reported worsening of chest pain 45 hours following the block, when a first rescue analgesia was requested. Symptoms improved after iv tramadol. Due to family reasons, the patient was transferred in another hospital after 5 days, without any further complication.

\section{Discussion}

Which is the ideal anesthetic and the volume to be used in SPB, has yet to be determined. In the current literature, Authors have performed SPB using a variety of local anesthetics, most

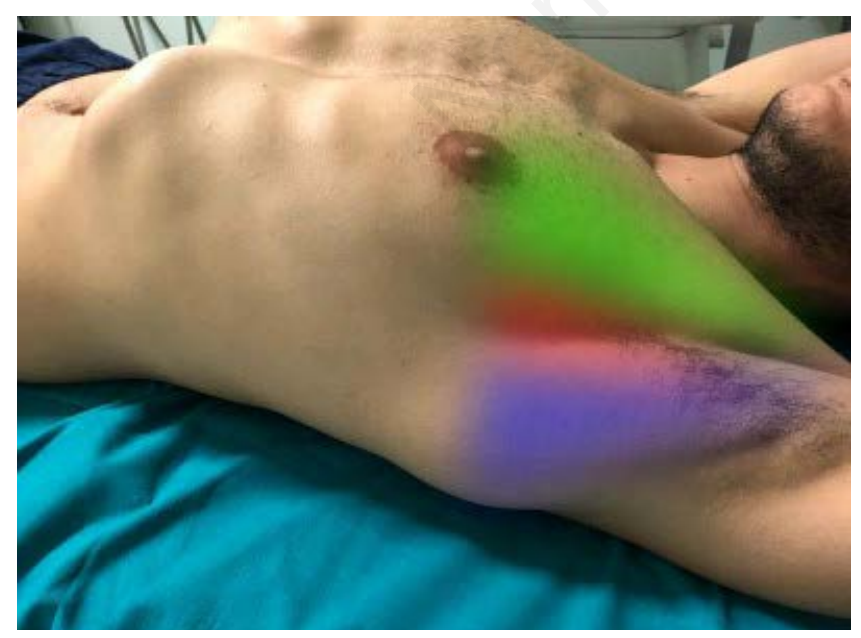

Figure 1. The anatomical landmarks for serratus block include the pectoralis major (green) and the latissimus dorsi (blue). In between these two muscles, the serratus muscle can be found on the mid-axillary line (red). At this level, we placed the linear sonographic probe in a transverse fashion. commonly ropivacaine, lidocaine or levobupivacaine, at different concentrations and volumes. In the preliminary study conducted on healthy volunteers by Blanco et al., ${ }^{1}$ they injected $0.4 \mathrm{ml} . \mathrm{kg}^{-1}$ $0.125 \%$ levobupivacaine in the fascial plane below and above the serratus muscle, diluted with gadolinium for research-related imaging purposes, followed by paresthesia lasting 12.5 to 14 hours. In addition to this, they also noted a wider dermatomal distribution and a longer duration of paresthesia when the injection was made in the fascial plane superficial to serratus muscle. In the setting of rib fractures, Durant et al..$^{5}$ obtained pain control for 1012 hours in two subjects with multiple rib fractures, by using $30 \mathrm{ml}$ of $0.5 \%$ ropivacaine supplemented with $3 \mathrm{ml}$ of $1 \%$ lidocaine. Similarly, Kunhabdulla et al. ${ }^{6}$ described pain control lasting 4-8 hours using $20 \mathrm{ml}$ of $0.125 \%$ bupivacaine for SPB in a single patient with multiple rib fractures, followed by continuous infusion of $0.0625 \%$ bupivacaine once the effects of the single-shot block ceased. A recent randomized clinical trial conducted on patients undergoing breast cancer surgery, suggests that using $40 \mathrm{~mL}$ of $0.375 \%$ ropivacaine instead of $20 \mathrm{~mL}$, provides a larger craniocaudal spread of the anesthetic, resulting in a greater number of affected dermatomes. ${ }^{7}$ However, the use of a larger quantity of anesthetic didn't show any advantage in terms of duration of pain control. ${ }^{7}$

To our knowledge, no previous study documented the addition of dexamethasone to the local anesthetic in SPB, which in principle could lead to a prolonged duration of the block. ${ }^{8}$

In our limited experience, the use of $30 \mathrm{~mL}$ of $0.25 \%$ levobupivacaine diluted with $8 \mathrm{mg}$ dexamethasone provided pain control lasting 23 to 45 hours in patients with multiple rib fractures. Such a long-lasting effect can be explained by two main factors: first of all, levobupivacaine is a more potent anesthetic for peripheral nerve block as compared to ropivacaine. ${ }^{9}$ Secondly, dexametha-

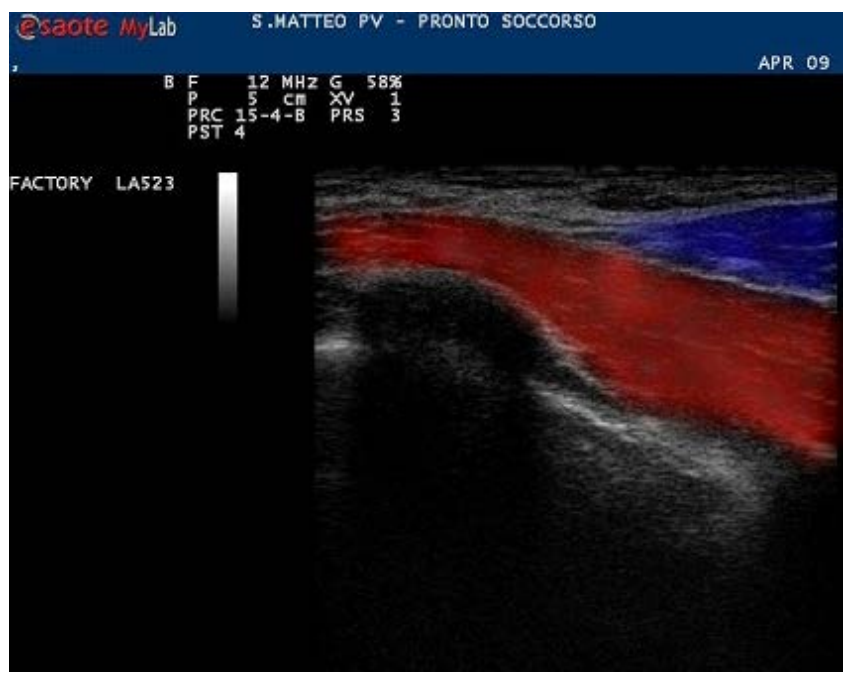

Figure 2. In the sonographic image obtained, the rib can be easily identified due to the acoustic shadowing, as well as the hyperechoic pleural line beneath it. The latissimus dorsi (blue) lies posterior and more superficial to the serratus muscle (red), whereas the pectoralis major is found more anteriorly and cannot be visualized in this image. Following identification of all the relevant landmarks, we inserted the needle in-plane from anterior to posterior, targeting the fascial plane superficial to the serratus muscle. The anterior to posterior approach was made necessary by the fact that all our patients were unable to turn on their flank, being therefore forced in the supine position. 
sone administered either intravenous or perineural is well known to further prolong the duration of nerve blocks. ${ }^{8}$ The prolonged effect of the block obtained in our patients, allowed to limit the use of opioids, that were even not deemed necessary in one case. Despite all three patients experienced breathing difficulties due to pain, they were able to maintain an adequate ventilation even before the block, therefore we did not report any improvement in terms of oxygen saturation following the procedure. However, what we observed indeed was a reduction of patient's respiratory workload, which decreased progressively with the onset of local anesthetic.

In principle, SPB can be repeated several times in case of pain recurrence, although in our experience this is usually unnecessary: in fact, rib fracture pain typically reaches its apex in the very first days following the trauma, after which it can usually be treated with NSAIDs and paracetamol. Therefore, we suggest to tackle rib fracture pain especially in the acute setting, with a multimodal therapy that should include locoregional analgesia. Of course, even though SPB can help reduce the use of opioids in rib fractures, patients may still require them especially if they have concomitant lesions other than rib fractures (see case n. 3). We reported no complications or adverse effects related to SPB. Moreover, none of the three patients developed respiratory failure or pneumonia during their hospital stay.

\section{Conclusions}

In conclusion, SPB confirmed to be an effective and easy to perform technique for pain control in multiple rib fractures. In our experience, levobupivacaine combined with dexamethasone guarantees a prolonged analgesia as compared to the evidence reported by other authors, though this finding needs to be confirmed in a randomized clinical trial.

\section{References}

1. Blanco R, Parras T, McDonnell JG, Prats-Galino A. Serratus plane block: a novel ultrasound-guided thoracic wall nerve block. Anaesthesia 2013;68:1107-13.

2. Piraccini E, Calli M, Byrne H, et al. Ultrasound-guided pectoral nerves and serratus plane block for post thoracotomy pain syndrome. Minerva Anestesiol 2017;83:888-9.

3. Hironobu U, Wataru I, Hiroshi O. Serratus plane block for a contraction of the latissimus dorsi muscle. regional anesthesia and pain medicine. Reg Anesth Pain Med 2016;41:411.

4. Piracha MM, Thorp SL, Puttanniah V, Gulati A. "A Tale of Two Planes" deep versus superficial serratus plane block for postmastectomy pain syndrome. Reg Anesth Pain Med 2017;42:259-62.

5. Durant E, Dixon B, Luftig J. Ultrasound-guided serratus plane block for ED rib fracture pain control. Am J Emerg Med 2017;35:197.e3-.e6.

6. Kunhabdulla NP, Agarwal A, Gaur A, et al. Serratus anterior plane block for multiple rib fractures. Pain Physician 2014;17:E553-5.

7. Kunigo T, Murouchi T, Yamamoto S, et al. Injection volume and anesthetic effect in serratus plane block. Reg Anesth Pain Med 2017;42:6.

8. Pehora C, Pearson AME, Kaushal A, et al. Dexamethasone as an adjuvant to peripheral nerve block. Cochrane Database System Rev 2017;11:CD011770.

9. Li A, Wei Z, Liu Y, et al. Ropivacaine versus levobupivacaine in peripheral nerve block. A PRISMA-compliant meta-analyses of randomized controlled trials. Medicine (Baltimore) 2017;96:e6551. 